\title{
Der Einfluss positiver und negativer Stimmungen von Befragten auf ihr Antwortverhalten in politischen Meinungsumfragen
}

\author{
Sylvia Kämpfer / Michael Mutz
}

The Influence of Positive and Negative Mood on Political Judgments in Public Opinion Polls Abstract: Empirical research in social and political science is fundamentally based on survey data. In these surveys respondents are usually asked for judgments and opinions about political issues, e. g. their satisfaction with the government or the evaluation of the state of the economy. This paper argues that these judgments do not solely rely on rational considerations of the issue to be assessed, but also reflect the mood experienced by the interviewee. Using the German samples of the European Social Surveys 2006 and 2012 it can be demonstrated that many of the indicators frequently used in political opinion polls are strongly influenced by the affective state of the respondent in the days preceding the interview. As expected, a positive mood lead to more positive judgments on political issues while negative affective states result in more negative judgments. Further analyses indicate that these effects are more pronounced in those respondents who are not thoroughly informed about political issues and spend only little time to catch up with current affairs.

Keywords: Public opinion, survey research, reliability, emotion, mood

Schlagwörter: Öffentliche Meinung, Umfrageforschung, Reliabilität, Emotion, Stimmung

\section{Einleitung}

Urteile über die Arbeit der Bundesregierung oder über einzelne Politiker, Einschätzungen der allgemeinen Wirtschaftslage oder Fragen zum Vertrauen der Bürgerinnen und Bürger in politische Institutionen sind fester Bestandteil politischer Umfragen, die in regelmäßigen Abständen von Meinungsforschungsinstituten durchgeführt werden. Die Befunde solcher Meinungsumfragen stoßen dabei auf ein allgemeines Interesse: Sie werden von den politischen Eliten zur Kenntnis genommen, von Journalisten als Anhaltspunkte für die aktuelle „politische Stimmung “ im Land interpretiert, und sie erreichen, vermittelt über Medien, eine breite Öffentlichkeit - man denke nur an Formate wie den ARD Deutschland-Trend oder das ZDF Politikbarometer. Darüber hinaus stützen sich aber auch zahlreiche wissenschaftliche Analysen auf politische Einschätzungen der Bürgerinnen und Bürger, die über Umfragen erhoben und als Indikatoren für grundlegende Einstellungen zum politischen System sowie als zentrale Handlungsprädiktoren verwendet werden. Dazu zählen u. a. Studien zum Wahlverhalten (vgl. u. a. Kellermann u. Rattinger 2007; Pappi u. Bytzek 2007; Rattinger u. Faas 2001; Schoen 2010) ebenso wie Arbeiten zur politischen Kultur- und Demokratieforschung (vgl. u. a. Freitag u. Bühlmann 2005; Fuchs et al. 2002; de Rijke et al. 2006; Westle u. Niedermayer 2009). Überblicksbeiträge zur Datennutzung in der empirischen Wahl- 
und Politikforschung sowie der politischen Soziologie weisen übereinstimmend darauf hin, dass quantitative Umfragedaten die wichtigste und am häufigsten genutzte Datengrundlage darstellen (Gabriel u. Maier 2009; Schoen 2005).

Gerade vor diesem Hintergrund gehört die kritische Reflexion der in der quantitativen Einstellungs- und Meinungsforschung verwendeten Indikatoren zu den Aufgaben, mit denen sich die empirische Politikwissenschaft auseinandersetzen muss. Diese Reflexion der eigenen Methoden dient nicht zuletzt dem Zweck, mögliche Quellen für systematische Verzerrungen zu identifizieren und nach Möglichkeit zu beseitigen. Man denke an die bekannten systematischen Antwortverzerrungen die durch Fragebogeneffekte ausgelöst werden, auf sozialer Erwünschtheit oder Akquieszenz beruhen oder aus situativen Einflüssen der Befragungssituation resultieren, z. B. Zeitdruck des Befragten, Sympathie für den Interviewer (vgl. hierzu im Überblick u. a. Schnell et al. 2008, S. 353-357).

Anknüpfend an diese Forschungsbefunde befasst sich der Beitrag mit einer methodischen Frage, die auf die Reliabilität einiger zentraler Indikatoren der politikwissenschaftlichen Umfrageforschung abhebt. Es geht darum, zu begründen und empirisch zu bestimmen, ob bzw. inwieweit die Antworten auf politische Einschätzungsfragen von den Stimmungen der Befragten beeinflusst werden. In Befragungssituationen, in denen in kurzer Zeit die verschiedensten politischen Sachverhalte bewertet werden sollen, zu denen sich die Befragten womöglich noch keine Meinung gebildet haben, dürften die Interviewten bei ihren Einschätzungen keineswegs immer nur auf Informationen zurückgreifen, die unmittelbar mit dem zu bewertenden Sachverhalt in Zusammenhang stehen und damit für den Sachverhalt relevant und für dessen Bewertung repräsentativ sind. Vielmehr ist zu vermuten, dass Personen in Befragungen häufig spontan antworten und sich dabei unbewusst auf ihre Stimmungen ${ }^{1}$ stützen, die nichts mit den zu bewertenden Sachverhalten zu tun haben und diese in ihre Einschätzungen einfließen lassen. Der Beitrag analysiert, ob bzw. inwieweit dies tatsächlich der Fall ist.

Dazu wird zunächst auf theoretischer Ebene erläutert, wie Befragte zu ihrer individuellen Einschätzung eines (politischen) Sachverhaltes gelangen und welche Rolle ihre Stimmungen in diesem Einschätzungsprozess spielen. Dabei wird im Rekurs auf psychologische Theorien und Forschungserkenntnisse argumentiert, dass Stimmungen zum einen direkt als Informationen in den Einschätzungspro-

1 Wenn wir im Folgenden von Stimmungen sprechen, meinen wir streng genommen auch Emotionen und Körpergefühle. Stimmungen, Emotionen und Körpergefühle unterscheiden sich bezüglich ihres konkreten Auslösers: So sind Stimmungen meist ohne klaren Auslöser, also objektunabhängig, während Emotionen (wie Angst oder Wut) von konkreten situativen Stimuli und Körpergefühle (wie Hunger, Schmerz oder Müdigkeit) vom momentanen physiologischen Zustand des Organismus ausgelöst werden (Schwarz 2012; Gasper u. Clore 2000). Da es in diesem Beitrag aber um die Wirkung von affektiven Zuständen geht, die nicht von dem zu beurteilenden Sachverhalt ausgelöst werden, sondern die einen vom zu bewertenden Objekt oder Sachverhalt unabhängigen Auslöser haben, ist es u. E. vertretbar, auf eine sprachliche Differenzierung zu verzichten. Wir sprechen im Beitrag deshalb generell von Stimmungen, obgleich uns bewusst ist, dass die später verwendeten Indikatoren auch Emotionen und Körpergefühle einbeziehen. Davon abgesehen besitzen sowohl Stimmungen als auch Emotionen und Körpergefühle eine mehr oder weniger klare Valenz, d. h. sie werden von den Betroffenen als angenehm oder unangenehm empfunden, sodass sie sich in ihrem Einfluss auf das Antwortverhalten in politischen Meinungsumfragen nicht wesentlich unterscheiden dürften (Schwarz 2012). 
zess einbezogen werden und zum anderen indirekt beeinflussen, welche kognitiven Informationen Berücksichtigung finden. Zudem wird argumentiert, dass sich politisch weniger gut informierte Befragte stärker auf ihre zuvor erlebten Stimmungen bei der Einschätzung politischer Sachverhalte stützen und eher dazu neigen, die entsprechenden Einschätzungsfragen spontan und „aus dem Bauch heraus" zu beantworten. Anschließend wird der Einfluss der in den letzten Tagen empfundenen Stimmungen von Befragten auf ihre Einschätzungen verschiedener politischer Sachverhalte empirisch getestet. Dafür werden exemplarisch fünf zentrale Indikatoren aus der politikwissenschaftlichen Forschung herangezogen: die Einschätzung der Wirtschaftslage, der Regierungsarbeit und des Demokratiezustands sowie das Vertrauen in die Mitmenschen und in das nationale Parlament. Die Befunde zeigen, dass Stimmungen das Antwortverhalten wesentlich mitbestimmen, insbesondere bei Befragten, die politisch weniger gut informiert sind. Die Implikationen der Befunde werden im Fazit diskutiert.

\section{Stimmungen im Bewertungsprozess}

\subsection{Bewertung und Einschätzung als kognitive Prozesse}

Die Einschätzung eines Sachverhaltes, wie z. B. die Beurteilung der Regierungsarbeit oder der allgemeinen Wirtschaftslage, ist das Ergebnis eines mehr oder weniger komplexen kognitiven Prozesses, in welchem verschiedene Informationen über den jeweiligen Sachverhalt herangezogen und bewertet werden (Schwarz u. Strack 1999). Welche konkreten Informationen für die Einschätzung eines Sachverhaltes herangezogen werden, hängt dabei einerseits von der Zugänglichkeit der Informationen und andererseits von deren Repräsentativität bzw. Relevanz ab. Zugänglich sind Informationen, wenn sie aktuell wahrgenommen oder im Gedächtnis abgespeichert und zu einem bestimmten Zeitpunkt erinnert werden. ${ }^{2}$ Repräsentativ sind Informationen dagegen, wenn sie sich auf den zu bewertenden Sachverhalt beziehen, sie diesem also ursächlich zugeordnet werden können (Schwarz u. Strack 1999; Schimmack u. Oishi 2005). Sind ausreichend viele Informationen über den jeweiligen Sachverhalt gesammelt - wann das der Fall ist, hängt wiederum stark von der Person und ihrer Motivation ab (Chen et al. 1999) - müssen diese anschließend bewertet werden. Die Bewertung erfolgt dabei durch einen Vergleich der herangezogenen Informationen mit inneren oder äußeren Bewertungsstandards wie Erwartungen, Zielen oder Wertvorstellungen (Michalos 1985; Lucas u. Diener 2009). Der gleiche objektive Sachverhalt kann entsprechend unterschiedlich bewertet werden, je nachdem welche Informationen erinnert, welche der erinnerten Informationen als repräsentativ für den einzuschätzenden Sachverhalt beurteilt und mit welchen Bewertungsstandards diese Informationen verglichen werden.

2 Die Information kann über einen längeren Zeitraum hinweg zugänglich sein („,chronische Zugänglichkeit“) oder nur in einem bestimmten Moment („temporäre Zugänglichkeit“) (Schwarz u. Strack 1999). 
Dies macht die Bewertung eines Sachverhaltes grundsätzlich anfällig für zahlreiche Einflüsse, sodass ein Urteil nicht nur zwischen Personen variieren, sondern auch durch die Befragungssituation beeinflusst werden kann. Der Befragungssituation kommt vor allem dann eine besondere Bedeutung zu, wenn die Befragten keine bereits umfassend durchdachte Einschätzung zu einem Thema haben, auf die sie zurückgreifen können (Saris u. Sniderman 2004; Tourangeau et al. 2000). So zeigen verschiedene Studien, dass die Zugänglichkeit von Informationen auch durch die Interviewsituation selbst beeinflusst wird: Frageformulierung, Antwortvorgaben oder die Reihenfolge der verschiedenen Fragen verändern die Zugänglichkeit verschiedener Informationen und damit den Inhalt der Informationen, auf welchen die Bewertung letztlich beruht (Schwarz u. Strack 1991; Tourangeau u. Rasinski 1988). Zudem kann die Befragungssituation auch einen Einfluss auf die Bewertung der Repräsentativität bzw. Relevanz der Informationen haben und damit ebenfalls beeinflussen, auf welche Informationen bei der Einschätzung zurückgegriffen wird. Situationen, in denen die Befragten ausreichend Gelegenheit haben sowie motiviert werden, eine umfassende, korrekte Einschätzung abzugeben, begünstigen eine systematische Suche nach Informationen, die für den zu bewertenden Sachverhalt repräsentativ sind, während unmotivierte Befragte unter Zeitnot viel eher dazu geneigt sind, alle Informationen, die momentan zugänglich sind, in die Einschätzung einfließen zu lassen, unabhängig davon wie repräsentativ eine Information tatsächlich ist (Chen et al. 1999, Gasper u. Clore 2000).

\subsection{Bedeutung von Stimmungen im Einschätzungsprozess}

Obwohl es sich bei der Einschätzung eines objektiven Sachverhaltes um das Ergebnis eines kognitiven Prozesses handelt, können sich Stimmungen und Emotionen nicht unerheblich auf die Einschätzung eines Sachverhaltes auswirken. Dies ist insbesondere dann sinnvoll und sogar notwendig, wenn es sich bei den Stimmungen und Emotionen um Informationen handelt, die für den einzuschätzenden Sachverhalt repräsentativ bzw. relevant sind. So macht es durchaus Sinn, wenn zur Beurteilung der Sympathie einer Person auf affektive Informationen zurückgegriffen wird. Allerdings scheinen Stimmungen und Emotionen auch dann die Einschätzung nicht unwesentlich zu beeinflussen, wenn sie in keinem oder nur in einem losen Zusammenhang mit dem zu beurteilenden Sachverhalt stehen. In der Literatur spricht man dann von „misattribution“ oder „incidental attribution“ (Forgas 2008). Dabei gilt allgemein, dass positive Stimmungen die Einschätzung eines Sachverhaltes eher positiv „verzerren“, während negative Stimmungen zur negativeren Einschätzung des gleichen Sachverhalts führen (Blanchette u. Richards 2010; Forgas 2008; Mayer et al. 1992).

Solche „verzerrten“ stimmungskongruenten Einschätzungen konnten in zahlreichen Studien, in denen die Stimmung der Probanden bewusst manipuliert wurde, nachgewiesen werden. Beispielsweise zeigen verschiedene Experimente, dass Personen, die zunächst in positive Stimmung versetzt und anschließend nach der Beurteilung ihres Lebens befragt wurden, ihr Leben insgesamt positiver bewerten 
(Schwarz u. Clore 1983). ${ }^{3}$ Andere Befunde weisen zudem darauf hin, dass solch eine „verzerrte“ stimmungskongruente Bewertung nicht nur bezüglich der Beurteilung des eigenen Lebens, sondern auch bezüglich anderer Einschätzungen wie Risiko-, Gefahren- und Produktbewertungen, bei der Beurteilung von Politkern und sogar bei der Bewertung physikalischer Realitäten existiert. In einer Studie von Johnson und Tversky (1983) schätzten Probanden mit negativ induzierten Stimmungen die Wahrscheinlichkeit, an Krankheiten oder durch Naturkatastrophen zu sterben, wesentlich höher ein als Personen, die sich in einer neutralen Stimmung befanden. Befunde von Haase und Silbereisen (2011) legen nahe, dass die Gefahren, die mit riskanten Verhaltensweisen verbunden sind, z. B. mit Drogengebrauch, ungeschütztem Geschlechtsverkehr, Autofahren in angetrunkenem Zustand, von positiv gestimmten Jugendlichen geringer eingeschätzt werden als von Jugendlichen, die neutral oder negativ gestimmt sind. Yueng und Wyer (2004) zeigen, dass in positive Stimmung versetzte Konsumenten ein Produkt positiver bewerten als in negative Stimmung versetzte Konsumenten, unabhängig davon, welche kognitiven Informationen ihnen über das Produkt zur Verfügung standen (vgl. auch Adaval 2001; Gorn 1982; Pham 1998; Pham et al. 2001; Shiv u. Fedorikhin 1999). Isbell und Wyer (1999) sowie Ottati und Isbell (1996) fanden heraus, dass Politiker positiver bewertet wurden, wenn die Befragten glücklich waren und negativer, wenn sie traurig waren. Und schließlich zeigt Proffitt (2006), dass traurige und ängstliche Personen die Steigung eines vor ihnen liegenden Berges sowie die Distanz bis zum Gipfel des Berges größer einschätzen als neutral gestimmte Personen, wobei die Stimmungen durch traurige Musik und einen schweren Rucksack auf dem Rücken manipuliert wurden.

All diese Studien zeigen, dass sich die Stimmung einer Person zum Befragungszeitpunkt auf ihre Einschätzungen objektiver Sachverhalte auswirkt, die entsprechend stimmungskongruent verzerrt werden. Wie es zu diesem Einfluss kommt, wird in der Literatur dabei durch zwei Mechanismen erklärt: (1) Zum einen wird ein direkter Einfluss von Stimmungen postuliert: Stimmungen fungieren hier als Informationen, die unmittelbar in die Einschätzung eines objektiven Sachverhaltes einfließen. (2) Zum anderen wird ein indirekter Einfluss begründet: Stimmungen strukturieren hierbei den kognitiven Prozess der Urteilsbildung, indem sie beeinflussen, welche kognitiven Informationen über den zu beurteilenden Sachverhalt wahrgenommen und erinnert werden (vgl. auch Forgas 2008).

3 Probanden beurteilten ihr Leben besser, wenn sie zuvor einen Sieg ihres Nationalteams bei der Fußballweltmeisterschaft miterlebt hatten. Probanden, deren Nationalteam unentschieden spielte, waren dagegen kurz nach dem Spiel unzufriedener mit ihrem Leben. Befragte, die in einem dreckigen, überhitzten, stinkenden und lauten Raum interviewt wurden (und daher in schlechter Stimmung waren), gaben eine geringere Lebenszufriedenheit an, als Probanden, deren Befragung in einem gemütlichen, komfortablen Raum stattfand. Schließlich beurteilten auch Studenten, die kurz zuvor eine scheinbar vergessene Geldmünze am Kopiergerät der Universitätsbibliothek fanden, ihr Leben besser, als jene Studenten, denen dieses Glück nicht vergönnt war. 


\subsection{Direkter Einfluss von Stimmungen}

Entsprechend der Feelings-as-Information-Theorie können neben kognitiven Informationen (als mentale Repräsentationen der Wirklichkeit) auch Stimmungen, Emotionen und Körpergefühle als Informationen in den Einschätzungsprozess einfließen (Schwartz et al. 1987; Schwarz 2012; Clore u. Huntsinger 2007). Dabei folgt der Einbezug von Stimmungen, Emotionen und Körpergefühlen prinzipiell den gleichen Regeln wie der Einbezug kognitiver Informationen: die zum Zeitpunkt der Einschätzung vorhandenen Affekte werden auf ihre Repräsentativität bezüglich des einzuschätzenden Sachverhaltes beurteilt und schließlich als positiv oder negativ für den einzuschätzenden Sachverhalt bewertet. Anders als bei kognitiven Informationen lässt sich bei Stimmungen, Emotionen und Körpergefühlen aber deutlich schwerer einschätzen, inwieweit diese den einzuschätzenden Sachverhalt repräsentieren. Zumindest neigen Menschen dazu, ihre Stimmungen, Emotionen und Körpergefühle auch dann in die Bewertung eines Sachverhalts einfließen zu lassen, wenn sie nicht durch den einzuschätzenden Sachverhalt selbst ausgelöst wurden. Schwarz (2012) erklärt das wie folgt: Menschen fällt es leicht, ihre Stimmungen, Emotionen und Körpergefühle wahrzunehmen, denn diese sind durch das unmittelbare Erleben leicht zugänglich. Anders als kognitive Informationen müssen sie nicht erst erinnert werden. Zudem beinhalten affektive Zustände durch ihre Valenz unmittelbar eine Bewertung, während kognitive Informationen erst durch einen Vergleich mit inneren und äußeren Bewertungsstandards für den Befragten Informationswert erhalten. Stimmungen, Emotionen und Körpergefühle werden universell als angenehm oder unangenehm bewertet und können daher als positive oder negative Informationen in die Beurteilung eines Sachverhaltes leicht einfließen. Vergleichsweise schwer ist es aber, anzugeben, wovon genau ein affektiver Zustand ausgelöst wurde (Gasper u. Clore 2000). Menschen gehen deshalb gewöhnlich davon aus, dass die aktuellen Stimmungen und Emotionen mit den Sachverhalten zusammenhängen, auf die sich die eigene Aufmerksamkeit gerade richtet. Dementsprechend werden sie oft fälschlicherweise als repräsentative Informationen wahrgenommen, auch wenn sie in keinem Zusammenhang mit dem einzuschätzenden Sachverhalt stehen.

Allerdings ist der Einfluss von Stimmungen, Emotionen und Körpergefühlen auf Bewertungen und Einschätzungen nicht immer gleich stark ausgeprägt, sondern hängt von einer Reihe anderer Faktoren ab: Ihre Bedeutung vergrößert sich immer dann, wenn die Bewertungskriterien unklar sind, nur unzureichende Informationen über den zu bewertenden Sachverhalt vorliegen, der Sachverhalt von der jeweiligen Person bislang noch nicht bewertet wurde (also noch keine frühere Bewertung vorliegt, auf die zurückgegriffen werden kann) und die kognitiven $\mathrm{Ka}$ pazitäten des Befragten sowie seine Motivation, den Sachverhalt korrekt und umfassend zu bewerten, gering ist (Greifeneder et al. 2010; Greifenender u. Bless 2007; Rotliman u. Schwarz 1998; Schwarz u. Strack 1999; Schwarz 2012; Ottati 
u. Isbell 1996). ${ }^{4}$ Somit ist beispielsweise anzunehmen, dass der Einfluss von aktuellen Stimmungen, Emotionen und Körpergefühlen auf die Bewertung von Sachverhalten größer ausfällt, wenn komplexe und abstrakte Sachverhalte eingeschätzt werden sollen, über die eine Person wenig Vorwissen besitzt, nur wenig Zeit für die Bewertung zur Verfügung steht und sich aus der Einschätzung für die Person keine nennenswerten Folgen ergeben (Forgas 2008). In Befragungssituationen sind zumindest einige dieser Bedingungen gegeben: Die Befragten müssen (a) relativ spontan und ohne längere Vorbereitungszeit viele unterschiedliche Sachverhalte einschätzen; sie dürften (b) viele dieser Einschätzungsfragen zum ersten Mal präsentiert bekommen, sodass sie auf keine umfassend durchdachte Meinung zurückgreifen können und (c) aus ihrer Antwort ergeben sich für sie selbst keinerlei positive oder negative Konsequenzen, sodass die Motivation für eine umfassende Abwägung gering ist. Sind zusätzlich die erfragten Sachverhalte komplex und abstrakt, dürfte es daher sehr wahrscheinlich sein, dass Befragte ihre Stimmungen, Emotionen und Körpergefühle als Informationen in die Einschätzung direkt einfließen lassen.

\subsection{Indirekter Einfluss von Stimmungen}

Neben dem eben erläuterten direkten Einbezug von Stimmungen als Informationen in den Einschätzungsprozess, können Stimmungen aber auch moderieren, welche Informationen in den Einschätzungsprozess einfließen, indem sie einerseits die Wahrnehmung und andererseits die Erinnerung von bestimmten kognitiven und/oder affektiven Informationen steuern.

So konnten zahlreiche psychologische Studien zeigen, dass positive Stimmungen zu einer erhöhten Wahrnehmung von positiv bewerteten Informationen führen, während negative Stimmungen zu einer bevorzugten Wahrnehmung negativ bewerteter Informationen führen (Mogg u. Bradley 1998; Tamir et al. 2006; Tamir u. Robinson 2007; Robinson u. Compton 2008). Zudem zeigen Arbeiten zum sogenannten Affect-Priming-Theorem, dass die Zugänglichkeit von Gedächtnisinhalten mit den Stimmungen einer Person variiert. Experimentelle Studien von Erisen et al. (2012) zeigen beispielsweise, dass Personen, die für einige Millisekunden ein lachendes Gesicht präsentiert bekommen, anschließend häufiger positive Einstellungen zu verschiedenen politischen Themen äußern, während Personen, die ein trauriges Gesicht sehen, negative Gedanken äußern. Es wird argumentiert, dass Gedächtnisinhalte immer auch eine affektive Tönung besitzen und insbesondere solche Information leichter aktualisiert werden können, die zu dem aktuellen Erleben und Empfinden einer Person passen. Fühlen sich Personen gut, können sie

4 Insbesondere die kognitiven Kapazitäten sowie die Motivation der Befragten entscheiden dem „dual mode-Modell“ zufolge darüber, ob überhaupt bzw. wie ausgiebig nach geeigneten Informationen gesucht und wie kritisch ihre Repräsentativität beurteilt wird (Chen et al.1999; Chaiken u. Trope 1999). Laut dem „dual mode-Modell“ gibt es zwei Modi der Informationsverarbeitung, einen aufwändigen „systematischen“ Modus sowie einen sparsamen „heuristischen“ Modus. Da der Mensch prinzipiell dazu geneigt ist, den Einsatz seiner kognitiven Ressourcen möglichst gering zu halten, wählt er den systematischen Weg der Informationsverarbeitung nur, wenn dies aufgrund der Situation als notwendig erscheint. 
positiv eingefärbte Gedächtnisinhalte (z. B. freudige Ereignisse) häufiger und schneller erinnern, als wenn sie sich schlecht fühlen (z. B. Denny u. Hunt 1992; Isen 2008; MacLeod u. Campbell 1992; Mayer et al. 1992; Robinson 2004; Robinson u. Compton 2008). Diese Effekte wurden zum einen in klinischen Stichproben gezeigt - depressive Probanden erinnern sich z. B. häufiger an jene kurz zuvor präsentierten Wörter, die eine negative Valenz aufweisen. Sie wurden zum anderen aber auch an nicht-klinischen Stichproben gezeigt, wo Stimmungen und Emotionen vorab manipuliert wurden, z. B. indem die Probanden vor der Messung der Erinnerungsleistung aufgefordert wurden, entweder eine besonders glückliche oder eine besonders traurige Episode aus ihrem Leben zu berichten. Die Befundlage zeigt übereinstimmend, dass traurige, depressive Personen eher negative Informationen erinnern, während fröhliche, gut gelaunte Menschen die positiven Aspekte einer Situation oder eines Sachverhalts betonen. Gerade Informationen mit politischem Inhalt besitzen häufig für eine Person eine affektive Tönung und werden daher den sogenannten „hot cognitions“ zugerechnet (Lodge u. Taber 2005). ${ }^{5}$ Deshalb dürfte die Wahrnehmung und Erinnerung von politischen Informationen besonders stark von den aktuellen Affekten abhängen.

Damit können sich Stimmungen also auch indirekt auf die Einschätzung eines (politischen) Sachverhaltes auswirken, indem sie die Wahrnehmung und Erinnerung von kognitiven und/oder affektiven Informationen beeinflussen. Die stimmungskongruente Wahrnehmung und Erinnerung von Informationen führt jeweils dazu, dass positive Stimmungen den Einbezug von positiven Informationen und negative Stimmungen den Einbezug von negativen Informationen in den Prozess der Urteilsbildung fördern. Sollen in einer Umfragesituation also Aspekte der wirtschaftlichen und politischen Lage eingeschätzt werden, dürften sich die Befragten, je nach ihren aktuellen Stimmungen, entweder eher an die positiven oder eher an die negativen Aspekte der einzuschätzenden Sachverhalte erinnern und entsprechend stimmungskongruente Bewertungen treffen, wobei dies auf Befragte ohne bereits umfassend durchdachte Meinung zum entsprechenden Sachverhalt noch stärker zutreffen sollte.

\subsection{Aktuelle vs. zeitlich zurückliegende Stimmungen}

Sowohl der direkte als auch der indirekte (verzerrende) Einfluss von Stimmungen wurde vorrangig anhand von aktuellen, zum Befragungszeitpunkt vorhandenen Stimmungen untersucht. Allerdings ist es durchaus plausibel, dass auch die Stimmung der letzten Tage einen Einfluss auf die Einschätzung eines Sachverhaltes haben kann. Hinweise darauf finden sich in der Lebenszufriedenheitsforschung: Zahlreiche Studien zeigen, dass die Einschätzung des eigenen Lebens maßgeblich durch die kurz zuvor erlebten Stimmungen und Emotionen beeinflusst wird. In

5 Hierbei werden kognitive Informationen bereits zu einem früheren Zeitpunkt mit Stimmungen und Emotionen verknüpft und entsprechend abgespeichert. Werden diese Informationen später erinnert, werden sowohl kognitive als auch affektive Anteile aktiviert. „All political leaders, groups, issues, symbols, and ideas thought about and evaluated in the past become affectively charged - positively or negatively - and this affect is linked directly to the concept in long-term memory" (Lodge u. Taber 2005, S. 456). 
entsprechenden Studien werden die Häufigkeit und Intensität von positiven und negativen Stimmungen und Emotionen während der letzten Tage oder der letzten Wochen erhoben und als Prädiktor der Lebenszufriedenheit verwendet. Dabei zeigt sich ein positiver Zusammenhang zwischen zurückliegenden positiven Stimmungen und Emotionen (wie Freude, Stolz, Heiterkeit und Liebe) und Lebenszufriedenheit sowie ein negativer Zusammenhang zwischen zurückliegenden negativen Stimmungen und Emotionen (wie Ärger, Angst, Schuld, Sorge und Eifersucht) und Lebenszufriedenheit (Kuppens et al. 2008; Mutz u. Kämpfer 2013; Pilcher 1998; Rojas u. Veenhoven 2013; Schimmack et al. 2002; Suh et al. 1998; Yardley u. Rice 1991). Generalisiert man diese Befunde auf Einschätzungsfragen im Allgemeinen, ist anzunehmen, dass auch zeitlich zurückliegende Stimmungen als Informationen direkt in die Einschätzung einfließen. ${ }^{6}$

Zudem wäre es denkbar, dass sich zeitlich zurückliegende Stimmungen auch indirekt auf die Einschätzung auswirken, indem sie die Wahrnehmung und Erinnerung von Informationen über den einzuschätzenden Sachverhalt verändern. Personen, die in der Zeit vor der Befragung häufiger negativ gestimmt waren, sollten in dieser Zeit vor allem negative Informationen wahrgenommen, im Gedächtnis gespeichert und erinnert haben, während Personen, die in der Vergangenheit positiv gestimmt waren, eher positive Informationen wahrgenommen, abgespeichert und erinnert haben sollten. Zuvor gut gestimmte Personen sollten in der Befragungssituation somit leichter auf positive Informationen zurückgreifen können als zuvor schlecht gestimmte Personen und umgekehrt (Schimmack et al. 2002). Folglich können sich auch zeitlich etwas zurückliegende Stimmungen vermittelt über die erhöhte Zugänglichkeit von positiven vs. negativen Informationen indirekt auf die Einschätzung eines Sachverhaltes in der Befragungssituation auswirken. Und schließlich dürfte die kürzlich empfundene Stimmung auch mit der aktuellen Stimmung korreliert sein, sodass die vorherrschenden Stimmungen der letzten Woche durchaus auch als Indikator für die aktuelle Stimmung herangezogen werden können.

\subsection{Forschungsleitende Hypothesen}

Aus diesen Überlegungen ergeben sich drei Hypothesen, die für unsere empirische Analyse leitend sind. Zunächst ist, gestützt auf die beiden eben skizzierten Theoreme, zu erwarten, dass die Stimmungen in die Einschätzung politischer Sachverhalte in der Befragungssituation einfließen und so zu stimmungskongruenten Ergebnissen führen - und zwar auch, wenn die Stimmungen in keinem Zusammenhang mit dem zu beurteilenden Sachverhalt stehen. Daher:

a. Je eher ein Befragter positiv gestimmt war, umso positiver werden politische Sachverhalte in der Befragungssituation eingeschätzt.

6 Selbstverständlich ist die zurückliegende Stimmung für die Beurteilung des eigenen Lebens tatsächlich repräsentativ, während zurückliegende Stimmungen vermutlich weniger repräsentativ für andere Sachverhalte wie beispielsweise die politische Lage eines Landes sind. Allerdings gelten auch hier die Argumente des Feelings-as-Information-Theorems: Aufgrund ihrer leichten Zugänglichkeit und ihrer Valenz neigen Personen dazu, auch nicht-repräsentative affektive Informationen bei Bewertungen zu berücksichtigen. 
b. Je eher ein Befragter negativ gestimmt war, umso negativer werden politische Sachverhalte in der Befragungssituation eingeschätzt.

Darüber hinaus haben wir argumentiert, dass der verzerrende Einfluss von aktuellen und zeitlich zurückliegenden Stimmungen auf die Bewertung von Sachverhalten von einer Reihe von Faktoren abhängt, die zum einen in der Befragungssituation und zum anderen in den Eigenschaften des Befragten liegen. Der Einfluss von Stimmungen sollte u. a. größer ausfallen, wenn eine Person vor der Befragung noch nicht umfassend über den einzuschätzenden Sachverhalt nachgedacht und sich noch keine entsprechende Meinung gebildet hat. Dies sollte vor allem bei jenen Personen der Fall sein, die sich über politische Themen nicht regelmäßig informieren. Bei politisch gut informierten Befragten wäre indes zu erwarten, dass die Stimmungslage weniger stark auf die Antworten abfärbt. ${ }^{7}$

c. Der Einfluss positiver und negativer Stimmungen auf die Einschätzung politischer Sachverhalte in einer Befragung ist bei Personen, die politisch gut informiert sind, geringer als bei Personen, die sich weniger gut über politische Themen informieren.

Alle drei Hypothesen werden im Folgenden getestet. Die Daten und Einschätzungsfragen, die wir dafür heranziehen, stellt der folgende Abschnitt vor.

\section{Zur Untersuchung}

Unsere Analyse stützt sich auf die deutschen Stichproben aus dem European Social Survey der Jahre 2006 und 2012 (ESS 2006, 2012). Beim ESS handelt es sich um eine Mehrthemenbefragung, die sowohl Kernthemen, die in jeder Erhebungswelle abgefragt werden, als auch wellenspezifische Themenschwerpunkte umfasst. $\mathrm{Zu}$ den Kernfragen gehören dabei einige Einschätzungsfragen zur politischen und wirtschaftlichen Lage, die in der politikwissenschaftlichen Umfrageforschung oft eingesetzt werden und die im ersten Teil der Befragung gestellt wurden. Zudem beinhalten die für diesen Beitrag herangezogenen Wellen Fragen zu den erlebten Emotionen der Befragten, welche im Rahmen des Themenschwerpunkts „Lebenszufriedenheit" erhoben und im hinteren Teil der Befragung gestellt wurden. ${ }^{8}$ Die

7 Lodge und Taber (2005) können zeigen, dass Stimmungen gerade dann eine hohe Wahrscheinlichkeit haben, in die Einschätzung eines Sachverhaltes Eingang zu finden, wenn eine Person intensiv über den entsprechenden Sachverhalt nachdenkt. Dies widerspricht nicht unserer Hypothese. Im Gegensatz zu Lodge und Taber geht es uns nicht um Affekte, die während des intensiven Nachdenkens über politische Themen entstehen, sondern um die Stimmungen, die der Befragungssituation zeitlich vorgelagert sind. Es könnte durchaus möglich sein, dass Personen, die sich intensiv mit dem einzuschätzenden Sachverhalt auseinandersetzen, sich bei ihrem Urteil weniger stark von zeitlich vorgelagerten Stimmungen leiten lassen, sondern, wie Lodge und Taber annehmen, von Emotionen, die durch die Auseinandersetzung mit dem zu beurteilende Thema erst evoziert werden bzw. während der Auseinandersetzung mit dem zu beurteilenden Sachverhalt vorherrschend waren.

8 Die Reihenfolge der Abfrage ist für die Analyse nicht unerheblich: Da die Stimmung der Befragten erst nach den Einschätzungsfragen erhoben wurde, ist ausgeschlossen, dass die Abfrage der Stimmungen den Befragten erst ihren emotionalen Zustand bewusst gemacht hat und sich dadurch bedingt im weiteren Verlauf des Interviews entsprechende stimmungskongruente Antworttendenzen einstellen. 
Daten des ESS eignen sich damit sehr gut zur Untersuchung unserer Hypothesen. Die Stichproben von jeweils knapp 3000 Befragten basieren auf einer Zufallsauswahl aller Personen ab 15 Jahren, die in einem privaten Haushalt in Deutschland leben. Sie sind damit jeweils repräsentativ für die deutsche Bevölkerung ab 15 Jahren. Die Befragung erfolgte computerunterstützt mündlich im Haushalt der Befragten (CAPI) durch geschulte Interviewer. Personen in den ostdeutschen Ländern wurden überproportional häufig befragt, sodass Gewichtungsfaktoren bei der Analyse berücksichtigt werden müssen, die diese Disproportionalität ausgleichen. Weitere Angaben zur Stichprobe und zur Erhebung sind auf der Webseite des ESS (http://www.europeansocialsurvey.de) dokumentiert.

(1) Als zu erklärende Variablen greifen wir auf fünf unterschiedliche Einschätzungsfragen zurück, die in der sozial- und politikwissenschaftlichen Forschung etabliert sind:

a. die Zufriedenheit mit der allgemeinen Wirtschaftslage im Land („Wie zufrieden sind Sie - alles in allem - mit der gegenwärtigen Wirtschaftslage in Deutschland?");

b. die Zufriedenheit mit der Arbeit der Bundesregierung („,Wenn Sie nun einmal an die Leistungen der Bundesregierung in Berlin denken. Wie zufrieden sind Sie mit der Art und Weise, wie sie ihre Arbeit erledigt? “);

c. die Zufriedenheit mit dem Zustand der Demokratie („Wie zufrieden sind Sie - alles in allem - mit der Art und Weise, wie die Demokratie in Deutschland funktioniert? “);

d. das Vertrauen in andere Menschen („Ganz allgemein gesprochen: Glauben Sie, dass man den meisten Menschen vertrauen kann, oder dass man im Umgang mit anderen Menschen nicht vorsichtig genug sein kann?“)

e. das Vertrauen in das Parlament („Sagen Sie mir zu jeder öffentlichen Einrichtung, die ich Ihnen nenne, wie sehr Sie persönlich jeder einzelnen davon vertrauen. [...] Bundestag?").

Bei jeder der fünf Fragen standen den Befragten Antwortmöglichkeiten auf einer 11-stufigen Ratingskala zur Verfügung. Bei den ersten drei Einschätzungen (Items a, b, c) reichten die Antwortvorgaben von (0) „äußerst unzufrieden“ bis (10) „äußerst zufrieden“. Bei der Frage zu sozialem Vertrauen (Item d) konnte die Antwort zwischen (0) „man kann nicht vorsichtig genug sein“ bis (10) „den meisten Menschen kann man vertrauen " variiert werden. Das Vertrauen in das Parlament konnte zwischen (0) „,vertraue überhaupt nicht“ und (10) „vertraue voll und ganz" abgestuft werden.

(2) Die zentralen Erklärungsvariablen für unsere Frage sind die erlebte positive und negative Stimmung. Um diese zu ermitteln, wurden die Personen befragt, wie oft sie in der letzten Woche verschiedene Affekte erlebt hatten.

a. Aus den Häufigkeiten, mit denen die Befragten (a) glücklich waren, (b) das Leben genossen haben, (c) viel Energie hatten und (d) ruhig und gelassen waren, bilden wir eine Skala der positiven Stimmung (Cronbachs $\alpha=.71$ ). 
b. Als negative Stimmung fassen wir die Häufigkeiten zusammen, mit denen sich die befragten Personen (a) deprimiert oder niedergeschlagen, (b) einsam und (c) traurig gefühlt haben sowie (d) ängstlich waren (Cronbachs $\alpha=.73) .{ }^{9}$

Beide Skalen basieren jeweils auf dem Mittelwert der vier Items. Sie können Werte zwischen 1 und 4 annehmen, wobei der Wert 1 für eine Person steht, die „nie oder fast nie " in der entsprechenden Stimmung war, während 4 eine Person beschreibt, die diese Stimmungen ,immer oder fast immer" erlebte. ${ }^{10}$

(3) In den Modellen müssen wir eine Reihe von Kontrollvariablen berücksichtigen. Zum einen kontrollieren wir soziodemografische und sozialstrukturelle Merkmale. Hierzu zählen (a) Alter, (b) Geschlecht, (c) Bildung (in Jahren), (d) Arbeitslosigkeit und (e) das Haushaltseinkommen. Zum anderen kontrollieren wir für Merkmale, die das allgemeine Interesse der Befragten an Politik abbilden. Hierzu gehört (f) die tägliche Zeit, die man zum Lesen von Zeitungsartikeln über Politik und aktuelle politische Ereignisse aufwendet, (g) die tägliche Zeit, in der man sich im Fernsehen Nachrichten oder Sendungen über Politik und aktuelle politische Ereignisse ansieht, (h) das selbst eingeschätzte Interesse für Politik, (i) die Bindung an eine der Regierungsparteien (2006: SPD, CDU/CSU; 2012: CDU/CSU, FDP) und (j) die Selbstpositionierung im politischen Links-Rechts-Spektrum als „links“ (Skalenwerte 0 bis 3) sowie als „rechts“ (Skalenwerte 7 bis 10). Die Kontrollvariablen sind im Online-Anhang zu diesem Beitrag genauer dokumentiert.

Diese Kontrollvariablen müssen aus zwei Gründen berücksichtigt werden: Verschiedene Bevölkerungsgruppen können zum einen auf systematisch unterschiedliche Art und Weise die abgefragten politischen Sachverhalte einschätzen bzw. von diesen betroffen sein. Arbeitslose, Geringverdiener, Ältere, aber auch Personen, die sich als sehr „links“ einschätzen, könnten die gegenwärtige Wirtschaftslage systematisch negativer beurteilen als der Bevölkerungsdurchschnitt. Zweitens sind auch positive und negative Stimmungen ungleich in der Bevölkerung verteilt: Arbeitslose empfinden z. B. häufiger Angst, während Selbständige und Personen in der oberen Dienstklasse öfter Ärger berichten (Rackow et al. 2012). Ohne Kontrolle dieser Einflüsse könnten die Effekte von Stimmungen auf die Bewertung politischer Sachverhalte systematisch über- oder unterschätzt werden.

(4) Schließlich testen wir einen Interaktionseffekt, den unsere dritte Hypothese postuliert. Hierfür haben wir die Befragten in zwei Gruppen eingeteilt und eine

9 Im ESS wurden noch weitere Emotionen, Stimmungen und Körpergefühle abgefragt (u. a. Müdigkeit oder Langeweile), die keine so eindeutige Valenz aufweisen, die also nicht so klar wie die einbezogenen Affekte als „positiv“ oder „negativ“ zugeordnet werden können. Die interne Konsistenz der Skalen würde sich zudem verschlechtern, wenn man diese weiteren affektiven Zustände berücksichtigt hätte.

10 Die Häufigkeit von positiven und negativen Stimmungen ist natürlich nicht vollkommen unabhängig voneinander, sondern korreliert miteinander. Die Korrelationen liegen knapp über $\mathrm{r}=.50$. Allerdings zeigt die psychologische Forschung, dass es sich bei positiven und negativen Affekten um zwei eigenständige Konstrukte und nicht bloß um zwei Seiten ein und derselben Medaille handelt. Es ist also durchaus möglich, in der letzten Woche gleichzeitig sehr häufig positive und negative Stimmungen erlebt zu haben (Diener et al. 1999). Wir haben deshalb auch alle acht abgefragten Affekte in eine Hauptkomponentenanalyse einbezogen. Diese identifiziert für beide ESS-Wellen zwei Faktoren mit Eigenwerten > 1, wobei die positiven Affekte deutlich stärker auf den ersten Faktor und die negativen Affekte stärker auf den zweiten Faktor laden. 
entsprechende Dummy-Variable gebildet. Jene Personen, die täglich mehr als 30 Minuten damit verbringen, Zeitungsartikel über Politik und aktuelle politische Ereignisse zu lesen, betrachten wir als politisch gut informiert $(\mathrm{N}=631)$. Alle anderen Befragten, die täglich weniger als 30 Minuten damit verbringen, entsprechende Zeitungsartikel zu lesen, betrachten wir als politisch weniger gut informiert $(\mathrm{N}=2281)$. Verknüpft man diese Dummy-Variable mit den erlebten positiven und negativen Stimmungen, so gibt dieser Interaktionsterm an, ob die Gruppe der politisch gut informierten Befragten ihre Einschätzungen weniger auf ihre Stimmungen stützt, als dies die Gruppe der weniger gut informierten Befragten macht. Da allerdings nur in der ESS-Welle 2006 die politische Zeitungslektüre erhoben wurde, können wir diesen Interaktionseffekt nur an einem der beiden Datensätze demonstrieren.

\section{Ergebnisse}

Die berechneten linearen Regressionsanalysen sind in den Tabellen 1 bis 5 (im Anhang) dokumentiert. Im ersten Modell sind nur die Stimmungseffekte ausgewiesen (ohne Kontrollvariablen). Das zweite Modell, das wir im Folgenden kommentieren, berücksichtigt alle genannten Kontrollvariablen. Es gibt also den um mögliche Drittvariableneinflüsse bereinigten Effekt für positive und negative Stimmung auf die Einschätzung politischer Sachverhalte an (Hypothese 1 und 2). Diese Modellierungen haben wir jeweils am ESS 2006 und 2012 durchgeführt. Ein drittes Modell, das nur mit den Daten des ESS 2006 berechnet werden kann, enthält zusätzlich die Interaktionsterme, mit deren Hilfe sich die Stimmungseffekte für politisch gut informierte Personen und für politisch weniger gut informierte Personen separat schätzen lassen. Diese Modellierung bezieht sich jeweils auf unsere dritte Hypothese.

\subsection{Einflüsse positiver und negativer Stimmungen auf politische Einschätzungen}

(1) Für die Bewertung der Arbeit der Bundesregierung zeigen sich in allen Modellvarianten erwartungskonforme, signifikante Effekte für positive und negative Stimmung. Auf der 11-stufigen Rating-Skala würde ein Befragter, der in der letzten Woche „immer oder fast immer“ positiv gestimmt war, die Bundesregierung im Durchschnitt mehr als einen Skalenpunkt besser bewerten als ein Befragter, der keine positiven Stimmungen berichtet (+1.14 Punkte [ESS 2006, Modell II]). ${ }^{11}$ Wer „immer oder fast immer“ negative Stimmung verspürte, schätzt die Bundesregierung um 0.78 Skalenpunkte schlechter ein, als ein Befragter, der nie negativ gestimmt war. Die Berechnungen am ESS 2012 ergeben ähnliche Befunde mit unwesentlich schwächeren Effekten. Im Vergleich mit den anderen unabhängigen Merkmalen ist die Stimmung ein starker Einflussfaktor für die Einschätzung der

11 Der angegebene Effekt ergibt sich bei einer Veränderung der Stimmungslage von 1 (= nie positive Stimmung) zu 4 (= immer oder fast immer positiv gestimmt). Multipliziert mit dem Regressionskoeffizienten $b$ (hier: 0.38) würde man bei einer entsprechenden Stimmungsänderung eine um 1.14 Skalenpunkte bessere Bewertung der Regierungsarbeit schätzen. Alle weiteren im Text angegebenen Effekte basieren auf identischen Überlegungen. 
Regierungsarbeit. Lediglich die Bindung an eine Regierungspartei strukturiert das Urteil noch etwas stärker.

(2) Die Einschätzung der gegenwärtigen wirtschaftlichen Lage ist sehr stark durch die Stimmung eingefärbt. In allen Modellen zeigen sich hypothesenkonforme Effekte für positive und negative Stimmung, die zudem sehr stark ausfallen. Befragte, die in der letzten Woche „immer oder fast immer“ positive Stimmungen erlebt haben, bewerten die Wirtschaftslage durchschnittlich etwa einen Skalenpunkt besser als Befragte, die keine positiven Stimmungen berichten (+1.02 Punkte [ESS 2006, Modell II]). Wer fast immer negative Stimmungen verspürte, schätzt die wirtschaftliche Lage sogar 1.59 Skalenpunkte schlechter ein als ein Befragter ohne negative Stimmungen. Die Regressionsmodelle mit den 2012 erhobenen ESS-Daten weisen auf geringfügig schwächere Effekte hin. Stimmungen färben aber auch in dieser Analyse signifikant und deutlich auf die Urteile über die Wirtschaftslage ab.

(3) Soll der Zustand der Demokratie beurteilt werden, zeigt sich abermals ein ähnliches Muster: Auch hier haben positive Stimmungen einen positiven Einfluss auf das Urteil, während negative Stimmungen mit skeptischeren Bewertungen einhergehen. Wer beispielsweise „immer oder fast immer“ positiv gestimmt war, bewertet den Zustand der Demokratie im Vergleich zu einer Person, die keine positiven Stimmungen angegeben hat, wiederum fast einen Skalenpunkt besser $(+0.96$ Punkte [ESS 2006, Modell II]). Wer in der Woche vor der Befragung immer in negativer Stimmung war, schätzt die Demokratie im Durchschnitt um 1.14 Skalenpunkte schlechter ein als eine Person, die keine negativen Stimmungen berichtet. Signifikante positive und negative Stimmungseffekte auf die Zufriedenheit mit der Demokratie sind auch in der ESS-Welle von 2012 zu erkennen. Die Hypothesen 1 und 2 werden auch hier bestätigt.

(4) Das soziale Vertrauen wird ebenfalls von der Stimmung beeinflusst, allerdings stärker von negativer Stimmung als von positiver Stimmung. Ein Befragter, der in der letzten Woche fast immer positive Stimmungen verspürt hat, äußert durchschnittlich nur ein leicht höheres Vertrauen in die Mitmenschen als ein Befragter, der keine positiven Stimmungen angibt $(+0.63$ Skalenpunkte [ESS 2006, Modell II]). Negative Stimmung führt hingegen zu deutlich negativeren Einschätzungen über die Mitmenschen: Wer „immer oder fast immer“ negativ gestimmt war, geht (im Vergleich zu einer Person ohne negative Stimmungen) im Durchschnitt deutlich eher davon aus, dass „man im Umgang mit anderen Menschen nicht vorsichtig genug sein kann“ (-1.35 Skalenpunkte [ESS 2006, Modell II]). Im ESS 2012 zeigt sich ein ähnlich starker negativer Einfluss von negativer Stimmung auf soziales Vertrauen. Vor allem negative Stimmung gehört mit zu den stärksten Prädiktoren für soziales Vertrauen.

(5) Schließlich können auch für die letzte der betrachteten Einschätzungsfragen, das Vertrauen in den Bundestag, die erwarteten Zusammenhänge im Wesentlichen bestätigt werden: Personen in zuletzt positiver Stimmung geben wiederum ein höheres Vertrauen und Personen in negativer Stimmung ein geringeres Vertrauen in das Parlament an. Wer durchweg positiv gestimmt war, liegt 0.69 Skalenpunkte über dem Vergleichswert einer Person ohne positive Gemütslage. Eine durchweg negativ gestimmte Person äußert dagegen im Durchschnitt 0.81 Punkte 
weniger Vertrauen ins Parlament (jeweils ESS 2006, Modell II). ${ }^{12}$ Im ESS 2012 lässt sich ein vergleichsweise starker Einfluss negativer Stimmung auf das Vertrauen in den Bundestag nachweisen. Positive Stimmungen haben allerdings keinen signifikanten positiven Effekt auf das entsprechende Urteil auf dem 95-ProzentSignifikanzniveau.

Insgesamt können die Modelle die Unterschiede in den Einschätzungen zu etwa 10 Prozent aufklären. ${ }^{13}$ Vor allem die Bindung an eine Regierungspartei, die Links-Rechts-Positionierung und die Zeitungslektüre politischer Nachrichten beeinflusst die Einschätzung politischer Sachverhalte meist signifikant. Soziodemografische Merkmale stehen nur zum Teil signifikant mit politischen Einschätzungen im Zusammenhang. Für die hier im Fokus stehenden Stimmungen zeigt sich aber eine sehr konsistente Befundlage: In allen Modellen fällt die Einschätzung der zu bewertenden Sachverhalte positiver aus, wenn die Befragten von positiver Stimmung berichten. Sie fällt umgekehrt negativer aus, wenn negative Stimmung angegeben wurde. Entsprechend können unsere ersten beiden Hypothesen bestätigt werden.

\subsection{Unterschiede zwischen politisch gut und politisch weniger gut informierten Befragten}

Wir haben argumentiert, dass in einer Befragungssituation vor allem dann mit verzerrten Antworttendenzen gerechnet werden muss, wenn sich die Personen über den einzuschätzenden Sachverhalt vor dem Zeitpunkt der Befragung noch keine Meinung gebildet haben. Personen ohne Meinung zu einem Thema geben eine spontane Antwort auf die ihnen gestellte Einschätzungsfrage und würden dabei - so die Hypothese - ihre Stimmung (unbewusst) stärker in die Antwort einfließen lassen. Wer sich umgekehrt bereits eine Meinung zu einem Thema gebildet hat, würde dagegen vornehmlich auf diese Meinung in der Umfragesituation zurückgreifen und seine Stimmung stärker ausblenden.

In der jeweiligen dritten Modellvariante (siehe Tabellen 1 bis 5) wird nun der Effekt der Stimmung für politisch gut informierte Personen und weniger gut informierte Personen separat geschätzt. ${ }^{14}$ Der Haupteffekt zeigt den Einfluss von positiven und negativen Stimmungen bei den Befragten an, die weniger gut politisch informiert sind, d. h. die angegeben haben, weniger als 30 Minuten am Tag

12 In weiteren, hier nicht dokumentierten Analysen zeigte sich, dass auch das Vertrauen in Politiker und in politische Parteien in ganz ähnlicher Weise von den Stimmungen des Befragten mitstrukturiert wird.

13 Der Anteil der aufgeklärten Varianz ist damit als gering zu interpretieren. Allerdings ist das für unsere Zielsetzung nicht weiter problematisch, da unser Beitrag nicht darauf abzielt, Unterschiede in den betrachteten Einschätzungen systematisch zu erklären und damit einen möglichst hohen Anteil der Varianz der abhängigen Variablen aufzuklären, sondern den spezifischen Einfluss von Stimmungen auf politische Urteile zu testen. Und hierfür ist es lediglich notwendig, jene Merkmale als Kontrollvariablen einzubeziehen, die mit der Stimmung von Menschen und mit der Ausprägung politischer Einstellungen korreliert sein könnten.

14 Grundsätzlich gibt es keine praktisch bedeutsamen Unterschiede in der Stimmungslage zwischen politisch gut informierten und politisch weniger gut informierten Personen. Die Korrelation von politischer Zeitungslektüre und positiven Stimmungen beträgt lediglich $r=.06$; die Korrelation mit negativen Stimmungen $r=-.05$. 
in die Lektüre politischer Nachrichten zu investieren. Addiert man zu diesem Haupteffekt den Interaktionseffekt hinzu, den wir im Modell III berechnen, erhält man die Schätzwerte für politisch gut informierte Personen, d. h. jene Befragten, die mindestens 30 Minuten am Tag mit der Lektüre politischer Nachrichten verbringen.

Die Ergebnisse zeigen zunächst fast ausnahmslos in die vermutete Richtung: Bei politisch gut informierten Personen hat der Interaktionseffekt für positive Stimmung ein negatives Vorzeichen. Bei der Addition von Haupt- und Interaktionseffekt ergibt sich nur noch ein geringer Einfluss der positiven Stimmung auf die Bewertung politischer Sachverhalte. Ähnliches gilt für negative Stimmung: Hier besitzt der Interaktionseffekt über alle abhängigen Variablen hinweg ein positives Vorzeichen, d. h. negative Stimmung wirkt sich bei politisch besser informierten Befragten weniger stark auf die Einschätzungen aus als bei politisch schlechter informierten Befragten. Kurzum: Wer pro Tag mindestens 30 Minuten in die Lektüre politischer Nachrichten investiert, lässt sich weitaus weniger in der Beurteilung der Regierungsarbeit, der Wirtschaftslage und der Demokratie von seiner Stimmung lenken und auch das Vertrauen in die Mitmenschen und in das Parlament hängt in deutlich geringerem Maße von der individuellen Stimmungslage ab. Der Unterschied zwischen politisch gut informierten und politisch weniger gut informierten Personen geht bei neun von zehn Interaktionstermen in die erwartete Richtung. In vier Fällen ist der Effekt signifikant $(\mathrm{p}<.05)$, in weiteren vier Fällen wird das 95 -Prozent-Signifikanzniveau nur sehr knapp verfehlt $(\mathrm{p}<.10)$. Wir sehen deshalb auch unsere dritte Hypothese im Wesentlichen als bestätigt an.

\section{Resümee}

Im Anschluss an etablierte psychologische Theoreme war zu erwarten, dass Einschätzungen und Bewertungen von politischen Sachverhalten in Surveys auch von der zuvor erlebten Stimmung der Befragten beeinflusst werden. An fünf gut etablierten Indikatoren der politikwissenschaftlichen Umfrageforschung, die ein breites Spektrum an politisch relevanten Sachverhalten repräsentieren, können wir einen sehr konsistenten Befund demonstrieren: Die in der Woche vor der Befragung empfundene positive Stimmung führt in der Befragungssituation zur positiveren Bewertungen politischer Sachverhalte, während die zuvor erlebten negativen Stimmungen negativere Bewertungen nach sich ziehen.

Vor allem die Stärke mit der Stimmungen in politische Einschätzungen einfließen, ist bemerkenswert: Bei den hier betrachteten Bewertungen, bei denen die Befragten ihre Antwort auf einer 11-stufigen Ratingskala abstufen sollten, liegen Befragte mit maximalen positiven Stimmungen und Befragte mit maximalen negativen Stimmungen teilweise um mehr als 2.5 Skalenpunkte auseinander. Das sind keineswegs marginale, sondern durchaus substanzielle Unterschiede. Diese Unterschiede zeigen sich, obwohl umfangreiche Kontrollvariablen berücksichtigt wurden. Besonders stark sind diese Stimmungseffekte bei der Frage nach der Wirtschaftslage, aber auch bei der Zufriedenheit mit der Demokratie und dem Vertrauen in die Mitmenschen. Zudem wurde deutlich, dass der Einfluss der Stimmung bei den Befragten größer ausfällt, die sich weniger umfassend politisch informieren, 
die also vermutlich in der Befragungssituation nicht auf eine im Vorfeld bereits gebildete Meinung zurückgreifen können, sondern spontane Antworten abgeben. Es ist davon auszugehen, dass die Stimmungseffekte wohl noch größer ausfallen würden, wenn wir nicht nur die vorherrschende Stimmung der letzten Tage, sondern auch die Stimmung zum Befragungszeitpunkt hätten einbeziehen können. Diese Informationen wurden im ESS aber leider nicht erhoben.

Was bedeutet das nun für die politikwissenschaftliche Umfrageforschung? Wir halten vier Schlussfolgerungen für relevant, die sich aus unserer Analyse ergeben:

(1) Häufig werden die hier analysierten Einschätzungsfragen für deskriptive Analysen verwendet. Dabei geht es darum, anhand von Durchschnittswerten und im Vergleich zu früheren Zeitpunkten Aussagen über die momentane „politische Stimmung “ im Land zu treffen. Für solche Deskriptionen sind die Implikationen, die sich aus unserer Analyse ergeben, weniger gravierend. Man kann nämlich davon ausgehen, dass die Stimmungen in der Bevölkerung normalerweise nicht kollektiv in die gleiche Richtung tendieren, sodass manche Befragte zum Befragungszeitpunkt positiv, andere hingegen negativ gestimmt sind. Manche Antworten sind daher positiv, andere negativ verzerrt. Die positiven und negativen Verzerrungen, die sich aus den erlebten Stimmungen ergeben, gleichen sich dann (mehr oder weniger) aus, sodass der Mittelwert im besten Fall nicht verzerrt ist.

(2) Zugleich stellt sich aber die Frage, ob es nicht doch Kollektivereignisse gibt, welche die Stimmungen größerer Bevölkerungsgruppen in die gleiche Richtung lenken könnten. Studien deuten beispielsweise darauf hin, dass besonders schönes und schlechtes Wetter die Gefühlslage von Personen beeinflussen und insofern auch die Antworten in Umfragen positiv oder negativ verzerren kann (Kämpfer u. Mutz 2013; Mutz u. Kämpfer 2011). Welche weiteren Ereignisse Stimmungen großer Bevölkerungsgruppen in die gleiche Richtung lenken, muss an dieser Stelle Spekulation bleiben. Man kann aber nicht ausschließen, dass bedeutende Ereignisse, etwa Terroranschläge, Todesfälle besonders prominenter und beliebter Personen oder Erfolge bei Fußballweltmeisterschaften oder ähnlich populären Sportereignissen, die Stimmung einer Bevölkerung kollektiv in eine bestimmte Richtung lenken können. Allerdings sind das Ereignisse, die nur ausgesprochen selten auftreten.

(3) Denkt man nicht an deskriptive, sondern an explanative Analysen, erscheint es sinnvoll, Fragen zu den Stimmungen und Emotionen der Befragten systematisch in Surveys zu integrieren, um diese bei späteren Auswertungen als Kontrollvariablen zur Verfügung zu haben. Falls nämlich bestimmte Stimmungen und Emotionen (wie Ärger oder Angst) in einigen Bevölkerungsgruppen überproportional häufig auftreten, können Zusammenhänge systematisch verzerrt sein, wenn diese nicht berücksichtigt werden. Wenn beispielsweise Arbeitslose die Regierung schlechter bewerten, kann das einerseits daran liegen, dass die (Arbeitsmarkt-) Politik der Regierung tatsächlich in dieser Bevölkerungsgruppe besonders negativ bewertet wird. Andererseits wäre es möglich, dass Arbeitslose im Alltag häufiger Negatives erleben, eher negative Stimmungen verspüren und deshalb negativ eingefärbte Antworten geben. Nur durch Einbezug von Stimmungen und Emotionen in die Analyse lassen sich solche Effekte voneinander unterscheiden.

(4) Schließlich legen unsere Befunde nahe, in Umfragen möglichst auf einfache und konkrete Fragen zurückzugreifen. Selbst Sozialwissenschaftler müssten wohl 
erst einige Überlegungszeit investieren, um umfassend abzuwägen, in welchem Zustand eigentlich die Demokratie in Deutschland ist oder wie die Wirtschaft sich wohl entwickeln wird. Für andere Bevölkerungsgruppen dürften diese Einschätzungsfragen noch abstrakter, komplexer und alltagsferner wirken. Die Befragten, die vor dem Befragungszeitpunkt noch nicht intensiv über den einzuschätzenden Sachverhalt nachgedacht und sich noch keine Meinung zu dem entsprechenden Thema gebildet haben, dürften bei solch schwierigen Bewertungen eher spontan „aus dem Bauch heraus“ antworten und dabei auch die Stimmung einfließen lassen. 15

Es dürfte aber auch Möglichkeiten geben, stimmungskongruente Antwortverzerrungen bereits während der Befragungssituation zu vermeiden: Grundsätzlich sind Personen darum bemüht, nur solche Informationen in die Bewertung einfließen zu lassen, die mit dem zu bewertenden Sachverhalt zusammenhängen bzw. von dem zu bewertenden Sachverhalt selbst ausgelöst wurden. Daher beziehen Personen nicht-repräsentative Stimmungen und Emotionen nur dann in die Einschätzung eines Sachverhaltes ein, wenn den Personen diese Fehlattribution selbst nicht bewusst ist und sie nicht darauf hingewiesen werden. Sobald eine Person aber darauf aufmerksam gemacht wird, dass ihre Stimmungen durch etwas anderes als durch den zu bewertenden Sachverhalt ausgelöst wurden, sucht sie nach anderen Informationen, die den Sachverhalt besser repräsentieren (Schwarz 2012; Clore u. Huntsinger 2007).

\section{Literatur}

Adaval, Rashmi. 2001. Sometimes It Just Feels Right. The Differential Weighting of AffectConsistent and Affect-Inconsistent Product Information. Journal of Consumer Research 28: 1-17.

Blanchette, Isabelle, und Anne Richards. 2010. The Influence of Affect on Higher Level Cognition. A Review of Research on Interpretation, Judgement, Decision Making and Reasoning. Cognition and Emotion 24: 561-595. doi: 10.1080/02699930903132496.

Chaiken, Shelly, und Yaacov Trope (Hrsg.) 1999. Dual-Process Theories in Social Psychology. New York: Guilford Press.

Chen, Serena, Kimberly Duckworth, und Shelly Chaiken. 1999. Motivated Heuristic and Systematic Processing. Psychological Inquiry 10: 44-49.

Clore, Gerald. L., und Jeffrey R. Huntsinger. 2007. How Emotions Inform Judgment and Regulate Thought. Trends in Cognitive Sciences 11: 393-399. doi: 10.1016/j. tics.2007.08.005.

Denny, Elizabeth.B., und R. Reed Hunt. 1992. Affective Valence and Memory in Depression: Dissociation of Recall and Fragment Completion. Journal of Abnormal Psychology 101: 575-580.

15 Stimmt diese Überlegung, dann dürfte unseren Befunden nach die Frage nach dem Vertrauen ins Parlament noch leichter zu beantworten sein als bspw. die Frage zur Zufriedenheit mit der gegenwärtigen Wirtschaftslage, denn die Stimmungseffekte auf die Antwort fallen im ersten Beispiel am geringsten und bei der zweiten Frage am stärksten aus. Explizit testen lässt sich das entsprechende Argument mit ESS-Daten aber nicht. 
de Rijke, Johann, Wolfgang Gaiser, Martina Gille, und Sabine Sardei-Biermann. 2006. Wandel der Einstellungen junger Menschen zur Demokratie in West- und Ostdeutschland. Ideal, Zufriedenheit, Kritik. Diskurs Kindheits- und Jugendforschung 1: 335-352.

Diener, Ed, Eunkook M. Suh, Richard E. Lucas, und Heidi L. Smith. 1999. Subjective WellBeing: Three Decades of Progress. Psychological Bulletin 125: 276-302.

Erisen, Cengiz., Milton Lodge, und Charles S. Taber. 2012. Affective Contagion in Effortful Political Thinking. Political Psychology. doi: 10.1111/j.1467-9221.2012.00937.x.

ESS European Social Survey Round 3 Data. 2006. Data file edition 3.4. Norwegen: Norwegian Social Science Data Services.

ESS European Social Survey Round 6 Data. 2012. Data file edition 1.1. Norwegen: Norwegian Social Science Data Services.

Forgas, Joseph P. 2008. Affect and Cognition. Perspectives on Psychological Science 3: 94101. doi: 10.1111/j.1745-6916.2008.00067.x.

Freitag, Markus, und Marc Bühlmann. 2005. Politische Institutionen und die Entwicklung generalisierten Vertrauens. Politische Vierteljahresschrift 46: 575-601.

Fuchs, Dieter, Oscar W. Gabriel, und Kerstin Völkl. 2002. Vertrauen in politische Institutionen und politische Unterstützung. Österreichische Zeitschrift für Politikwissenschaft 31: 427-450. PID: http://nbn-resolving.de/urn:nbn:de:0168-ssoar-60092.

Gabriel, Oscar W., und Jürgen Maier. 2009. Politische Soziologie in Deutschland. Forschungsfelder, Analyseperspektiven, ausgewählte empirische Befunde. Politische Vierteljahresschrift 50: 506-538.

Gasper, Karen, und Clore, Gerald. L. 2000. Do You Have to Pay Attention to Your Feelings in Order to be Influenced by Them? Personality and Social Psychology Bulletin 26: 698-711. doi: 10.1177/0146167200268005.

Gorn, Gerald J. 1982. The Effects of Music in Advertising on Choice Behavior. A Classical Conditioning Approach. Journal of Marketing 46: 94-101.

Greifeneder, Rainer, und Herbert Bless. 2007. Relying on Accessible Content versus Accessibility Experience. The Case of Processing Capacity. Social Cognition 25: 853-881. doi: 10.1521/soco.2007.25.6.853.

Greifeneder, Rainer, Herbert Bless, und Michel Tuan Pham. 2010. When Do People Rely on Affective and Cognitive Feelings in Judgment? A Review. Personality and Social Psychology Review 20: 1-35. doi: 10.1177/1088868310367640.

Haase, Claudia M., und Rainer K. Silbereisen. 2011. Effects of Positive Affect on Risk Perceptions in Adolescence and Young Adulthood. Journal of Adolescence 34: 29-37. doi: 10.1016/j.adolescence.2010.03.004.

Isbell, Linda M., und Robert S. Wyer. 1999. Correcting for Mood-Induced Bias in the Evaluation of Political Candidates. The Role of Intrinsic and Extrinsic Motivation. Personality and Social Psychology Bulletin 25: 237-249.

Isen, Alice M. 2008. Some Ways in Which Positive Affect Influences Decision Making and Problem Solving. In Handbook of Emotions, Hrsg. M. Lewis, J. M. Haviland-Jones und Lisa Feldman Barrett, 548-573. New York: Guilford Press.

Johnson, Eric M., und Amos Tversky. 1983. Affect, Generalization, and the Perception of Risk. Journal of Personality and Social Psychology 45: 20-31.

Kämpfer, Sylvia, und Michael Mutz. 2013. On the Sunny Side of Life. Weather Effects on Life Satisfaction. Social Indicators Research 110: 579-595. 
Kellermann, Charlotte, und Hans Rattinger. 2007. Wirtschaftslage, Arbeitslosigkeit und zugeschriebene Regierungsverantwortung als Bestimmungsfaktoren des Wahlverhaltens bei den Bundestagswahlen 2002 und 2005. In Die Bundestagswahl 2005: Analysen des Wahlkampfes und der Wablergebnisse, Hrsg. Frank Brettschneider, Oskar Niedermayer und Bernhard Weßels, 369-394. Wiesbaden: VS Verlag für Sozialwissenschaften.

Kuppens, Peter, Anu Realo, und Ed Diener. 2008. The Role of Positive and Negative Emotions in Life Satisfaction Judgment Across Nations. Journal of Personality and Social Psychology 95: 66-75. doi: 10.1037/0022-3514.95.1.66.

Lodge, Milton, und Charles. S. Taber. 2005. The Automaticity of Affect for Political Leaders, Groups, and Issues. An Experimental Test of the Hot Cognition Hypothesis. Political Psychology 26: 455-482. doi: 10.1111/j.1467-9221.2005.00426.x.

Lucas, Richard E., und Ed Diener. 2009. Personality and Subjective Well-Being. Social Indicators Research Series 37: 75-102.

MacLeod, Colin, und Lynee Campbell. 1992. Memory Accessibility and Probability Judgments. An Experimental Evaluation of the Availability Heuristic. Journal of Personality and Social Psychology 63: 890-902.

Mayer, John D.,Yvonne N. Gaschke, Debra L. Braverman, und Temperance W. Evans. 1992. Mood-Congruent Judgement Is a General Effect. Journal of Personality and Social Psychology 63: 119-132.

Michalos, Alex C. 1985. Multiple Discrepancies Theory. Social Indicators Research 16: 347-413.

Mogg, Karin, und Brendan P. Bradley. 1998. A Cognitive-motivational Analysis of Anxiety. Behaviour Research and Therapy 36: 809-848.

Mutz, Michael, und Sylvia Kämpfer. 2011....und nun zum Wetter. Beeinflusst die Wetterlage die Einschätzung von politischen und wirtschaftlichen Sachverhalten? Zeitschrift für Soziologie 40: 208-226.

Mutz, Michael, und Sylvia Kämpfer. 2013. Emotionen und Lebenszufriedenheit in der „Erlebnisgesellschaft“ - Eine vergleichende Analyse von 23 europäischen Ländern im Anschluss an die Gesellschaftsdiagnose von Gerhard Schulze. Kölner Zeitschrift für Soziologie und Sozialpsychologie 65: 253-275.

Ottati, Victor C., und Linda M. Isbell. 1996. Effects of Mood During Exposure to Target Information on Subsequently Reported Judgments. An On-Line Model of Misattribution and Correction. Journal of Personality and Social Psychology 71: 39-53.

Pappi, Franz Urban, und Evelyn Bytzek. 2007. Wirtschaftslage und die Popularität der Regierungen Schröder I und II. In Die Bundestagswahl 2005: Analysen des Wahlkampfes und der Wahlergebnisse, Hrsg. Frank Brettschneider, Oskar Niedermayer und Bernhard Wessels, 349-368. Wiesbaden: VS Verlag für Sozialwissenschaften.

Pham, Michel Tuan. 1998. Representativeness, Relevance and the Use of Feelings in Decision Making. Journal of Consumer Research 25: 144-60.

Pham, Michel Tuan, Joel B. Cohen, John W. Pracejus, und G. Davis Hughes. 2001. Affect Monitoring and the Primacy of Feelings in Judgment. Journal of Consumer Research 28: $167-88$.

Pilcher, June J. 1998. Affective and Daily Event Predictors of Life Satisfaction in College Students. Social Indicators Research 43: 291-306.

Proffitt, Dennis R. 2006. Embodied Perception and the Economy of Action. Perspectives on Psychological Science 1: 110-122. 
Rackow, Katja, Jürgen Schupp, und Christian von Scheve. 2012. Angst und Ärger. Zur Relevanz emotionaler Dimensionen sozialer Ungleichheit. Zeitschrift für Soziologie 41: 392-409.

Rattinger, Hans, und Thorsten Faas. 2001. Wahrnehmungen der Wirtschaftslage und Wahlverhalten 1977 bis 1998. In Wablen und Wäbler. Analysen aus Anlass der Bundestagswahl 1998, Hrsg. Hans-Dieter Klingemann und Max Kaase, 283-308. Wiesbaden: Westdeutscher Verlag.

Robinson, Michael D. 2004. Personality as Performance. Categorization Tendencies and Their Correlates. Current Directions in Psychological Science 13: 127-129.

Robinson, Michael D., und Rebecca J. Compton. 2008. The Happy Mind in Action: The Cognitive Basis of Subjective Well-Being. In The Science of Subjective Well-Being, Hrsg. Michael Eid und Randy J. Larsen, 220-238. New York: Guilford Press.

Rojas, Mariano, und Ruut Veenhoven. 2013. Contentment and Affect in the Estimation of Happiness. Social Indicators Research 110: 415-431.

Rotliman, Alexander J., und Norbert Schwarz. 1998. Constructing Perceptions of Vulnerability. Personal Relevance and the Use of Experiential Information in Health Judgments. Personality and Social Psychology Bulletin 24: 1053-1064. doi: 10.1177/01461672982410003.

Saris, Willem E., und Paul M. Sniderman. 2004. Studies in Public Opinion: Attitudes, Nonattitudes, Measurement Error, and Change. Princeton: Princeton University Press.

Schimmack, Ulrich, und Shigehiro Oishi. 2005. The Influence of Chronically and Temporarily Accessible Information on Life Satisfaction Judgments. Journal of Personality and Social Psychology 89: 395-406. doi: 10.1037/0022-3514.89.3.395.

Schimmack, Ulrich, Phanikiran Radhakrishnan, Shigehiro Oishi, Vivian Dzokoto, und Stephan Ahadi. 2002. Culture, Personality, and Subjective Well-being. Integrating Process Models of Life-satisfaction. Journal of Personality and Social Psychology 82: 582-593. Schnell, Rainer, Paul B. Hill, und Elke Esser. 2008. Methoden der empirischen Sozialforschung. München: Oldenbourg Verlag.

Schoen, Harald. 2005. Daten in der empirischen Wahlforschung. In Handbuch Wablforschung, Hrsg. Jürgen W. Falter und Harald Schoen, 89-103. Wiesbaden: VS Verlag für Sozialwissenschaften.

Schoen, Harald. 2010. Die Bürger ziehen Bilanz. Einstellungen zur Großen Koalition und Wahlverhalten 2009. In Die Große Koalition: Eine Bilanz der Regierung Merkel $2005-$ 2009, Hrsg. Christoph Egle und Reimut Zohlnhöfer, 29-50. Wiesbaden: VS Verlag für Sozialwissenschaften.

Schwarz, Norbert, Fritz Strack, Detlev Kommer, und Dirk Wagner. 1987. Soccer, Rooms, and the Quality of Life: Mood Effects on Judgements of Satisfaction with Life in General and with Specific Domains. European Journal of Social Psychology 17: 69-79. doi: 10.1002/ejsp.2420170107.

Schwarz, Norbert, und Gerald L. Clore. 1983. Mood, Misattribution, and Judgements of Well-being. Informative and Directive Functions of Affective States. Journal of Personality and Social Psychology 45: 513-523.

Schwarz, Norbert, und Fritz Strack. 1991. Context Effects in Attitude Surveys: Applying Cognitive Theory to Social Research. European Review of Social Psychology 2: 31-50. doi: 10.1080/14792779143000015. 
Schwarz, Norbert, und Fritz Strack. 1999. Reports of Subjective Well-being. Judgmental Processes and their Methodological Implications. In Well-Being: The Foundations of Hedonic Psychology, Hrsg. Daniel Kahnemann, Ed Diener und Norbert Schwarz, 6184. New York: Russell Sage Foundation.

Schwarz, Norbert. 2012. Feelings-as-Information Theory. In Handbook of Theories of Social Psychology, Hrsg. Paul van Lange, Arie W. Kruglanski und E. Tory Higgins, 289308. New York: Sage Publications.

Shiv, Baba, und Alexander Fedorikhin. 1999. Heart and Mind in Conflict. The Interplay of Affect and Cognition in Consumer Decision Making. Journal of Consumer Research 26: 278-92. doi: 10.1086/209563.

Suh, Eunkook, Ed Diener, Shigehiro Oishi, und Harry C. Triandis. 1998. The Shifting Basis of Life Satisfaction Judgments Across Cultures. Emotions Versus Norms. Journal of Personality and Social Psychology 74: 482-493.

Tamir, Maya, und Michael D. Robinson. 2007. The Happy Spotlight: Positive Mood and Selective Attention to Positive Information. Personality and Social Psychology Bulletin 33: 1124-1136.

Tamir, Maya, Michael D. Robinson, und Emily C. Solberg. 2006. You May Worry, But Can You Recognize Threats When You See Them? Neuroticism, Threat Identifications, and Negative Affect. Journal of Personality 74: 1481-1506. doi: 10.1111/j.1467-6494.2006. 00417.x.

Tourangeau, Roger, und Kenneth Rasinski. 1988. Cognitive Processes Underlying Context Effects in Attitude Measurement. Psychological Bulletin 103: 299-314.

Tourangeau, Roger, Lance J. Rips, und Kenneth Rasinski. 2000. The Psychology of Survey Response. New York: Cambridge University Press.

Westle, Bettina, und Oskar Niedermayer. 2009. Orientierungen gegenüber der Demokratie. In Wähler in Deutschland: sozialer und politischer Wandel, Gender und Wablverhalten, Hrsg. Steffen Kühnel, Oskar Niedermayer und Bettina Westle, 11-29. Wiesbaden: VS Verlag für Sozialwissenschaften.

Yardley, John K., und Robert W. Rice.1991. The Relationship between Mood and Subjective Well-Being. Social Indicators Research 24: 101-111.

Yueng, Catherine W. M., und Robert S. Wyer. 2004. Affect, Appraisal, and Consumer Judgment. Journal of Consumer Research 31: 412-424.

\section{Autorenangaben}

Dipl.-Soz. Sylvia Kämpfer

Freie Universität Berlin, Institut für Soziologie,

Garystr. 55,

14195 Berlin,

E-Mail: sylvia.kaempfer@fu-berlin.de

Prof. Dr. Michael Mutz

Georg-August-Universität Göttingen, Institut für Sportwissenschaften,

Sprangerweg 2,

37075 Göttingen,

michael.mutz@sport.uni-goettingen.de 


\section{Anhang}

\section{Tabelle 1: Einfluss individueller Stimmung auf die Bewertung der Regierungsarbeit}

\begin{tabular}{|c|c|c|c|c|c|c|c|c|}
\hline & \multicolumn{8}{|c|}{ Bewertung der Arbeit der Regierung } \\
\hline & ESS'06-I & \multicolumn{2}{|c|}{ ESS'06-II } & \multicolumn{2}{|c|}{ ESS’06-III } & ESS'12-I & \multicolumn{2}{|c|}{ ESS'12-II } \\
\hline & $\mathrm{b} \quad \beta$ & $\mathrm{b}$ & $\beta$ & $\mathrm{b}$ & $\beta$ & $\mathrm{b} \quad \beta$ & $\mathrm{b}$ & $\beta$ \\
\hline positive Stimmung ${ }^{1}$ & $0.43 * * .11$ & $0.38 * *$ & .10 & $0.41 * *$ & .11 & $0.43 * .12$ & $0.32 * *$ & .09 \\
\hline negative Stimmung ${ }^{1}$ & $-0.33^{* *}-.07$ & $-0.26^{*}$ & -.05 & $-0.31 * *$ & -.06 & $-0.21 *-.05$ & $-0.20 *$ & -.04 \\
\hline pos. Stimmg. *informiert ${ }^{1}$ & & & & $-0.15^{+}$ & -.08 & & & \\
\hline neg. Stimmg. ${ }^{*}$ informiert ${ }^{1}$ & & & & $0.31 *$ & .08 & & & \\
\hline Alter (in Jahren) & & -0.00 & -.03 & -0.00 & -.03 & & $-0.01 * *$ & -.09 \\
\hline Geschlecht (männlich) & & -0.01 & -.00 & -0.01 & -.00 & & $-0.23 * *$ & -.05 \\
\hline Bildung (in Jahren) & & $-0.03 *$ & -.05 & $-0.03 *$ & -.05 & & -0.00 & -.01 \\
\hline Arbeitslosigkeit & & -0.20 & -.02 & -0.20 & -.02 & & $-0.48 * *$ & -.05 \\
\hline HH.-Einkommen & & $0.04^{+}$ & .07 & $0.05^{+}$ & .07 & & $0.05 * *$ & .08 \\
\hline HH.-Eink.: k. Angabe & & 0.04 & .01 & 0.04 & .01 & & $0.37^{*}$ & .06 \\
\hline pol. Zeitungslektüre & & $0.28 * *$ & .10 & $0.27 * *$ & .10 & & & \\
\hline pol. TV-Konsum & & 0.04 & .02 & 0.03 & .02 & & -0.01 & -.01 \\
\hline pol. Interesse & & $0.13 *$ & .05 & $0.12 *$ & .05 & & 0.01 & .00 \\
\hline Bindung Reg.-Partei & & $0.71 * *$ & .15 & $0.71^{* *}$ & .15 & & $1.19 * *$ & .23 \\
\hline politisch „links“ & & $-0.42 * *$ & -.09 & $-0.42 * *$ & -.09 & & $-0.23 *$ & -.05 \\
\hline politisch „rechts“ & & 0.00 & .00 & 0.01 & .00 & & -0.03 & -.01 \\
\hline Modellgüte $\left(\mathrm{R}^{2}\right)$ & .025 & .076 & & .077 & & .024 & .100 & \\
\hline
\end{tabular}

Anmerkungen: Lineare Regressionsanalysen. $\mathrm{N}=2403$ (ESS 2006) und N = 2610 (ESS 2012). Signifikanzwerte: ${ }^{+} p<.10,{ }^{*} p<.05,{ }^{* *} p<.01$. 'Da gerichtete Zusammenhangshypothesen aufgestellt wurden, werden für die gekennzeichneten Indikatoren einseitige Signifikanztests verwendet. 
Tabelle 2: Einfluss individueller Stimmung auf die Bewertung der Wirtschaftslage

\begin{tabular}{|c|c|c|c|c|c|c|c|c|c|}
\hline & \multicolumn{9}{|c|}{ Bewertung der Lage der Wirtschaft } \\
\hline & ESS'06-I & \multicolumn{2}{|c|}{ ESS’06-II } & \multicolumn{2}{|c|}{ ESS’06-III } & \multicolumn{2}{|c|}{ ESS'12-I } & \multicolumn{2}{|c|}{ ESS'12-II } \\
\hline & $\mathrm{b} \quad \beta$ & $\mathrm{b}$ & $\beta$ & $\mathrm{b}$ & $\beta$ & $\mathrm{b}$ & $\beta$ & $\mathrm{b}$ & $\beta$ \\
\hline positive Stimmung $^{1}$ & $0.43^{* *} .11$ & $0.34 * *$ & .09 & $0.39 * *$ & .10 & $0.29 * *$ & .08 & $0.22 * *$ & .06 \\
\hline negative Stimmung ${ }^{1}$ & $-0.73^{* *}-.14$ & $-0.53 * *$ & -.10 & $-0.59 * *$ & -.11 & $-0.68 * *$ & -.15 & $-0.42 * *$ & -.09 \\
\hline pos. Stimmg. "informiert ${ }^{1}$ & & & & $-0.24 * *$ & -.13 & & & & \\
\hline neg. Stimmg. ${ }^{2}$ informiert ${ }^{1}$ & & & & $0.36^{* *}$ & .10 & & & & \\
\hline Alter (in Jahren) & & 0.00 & .00 & 0.00 & .01 & & & -0.00 & -.01 \\
\hline Geschlecht (männlich) & & 0.12 & .03 & 0.12 & .03 & & & $0.54 * *$ & .13 \\
\hline Bildung (in Jahren) & & $0.03 *$ & .04 & $0.03 *$ & .04 & & & $0.03 *$ & .05 \\
\hline Arbeitslosigkeit & & $-0.69 * *$ & -.08 & $-0.68 * *$ & -.08 & & & $-0.47 * *$ & -.05 \\
\hline HH.-Einkommen & & $0.13 * *$ & .19 & $0.13 * *$ & .19 & & & $0.09 * *$ & .14 \\
\hline HH.-Eink.: k. Angabe & & $0.62 * *$ & .12 & $0.64 * *$ & .12 & & & $0.61 * *$ & .10 \\
\hline pol. Zeitungslektüre & & $0.27 * *$ & .10 & $0.34 * *$ & .12 & & & & \\
\hline pol. TV-Konsum & & -0.06 & -.03 & -0.06 & -.03 & & & 0.02 & .01 \\
\hline pol. Interesse & & 0.06 & .02 & 0.05 & .02 & & & $-0.24 * *$ & -.09 \\
\hline Bindung Reg.-Partei & & $0.44 * *$ & .09 & $0.44 * *$ & .09 & & & $0.66^{* *}$ & .13 \\
\hline politisch „links“ & & $-0.36^{* *}$ & -.07 & $-0.36^{* *}$ & -.07 & & & -0.07 & -.02 \\
\hline politisch „rechts“ & & -0.03 & -.00 & -0.02 & -.00 & & & 0.17 & .03 \\
\hline Modellgüte $\left(\mathrm{R}^{2}\right)$ & .049 & .10 & & $.10 \varepsilon$ & & .042 & & .12 & \\
\hline
\end{tabular}

Anmerkungen: Lineare Regressionsanalysen. $\mathrm{N}=2433$ (ESS 2006) und N = 2639 (ESS 2012). Signifikanzwerte: ${ }^{+} p<.10,{ }^{*} p<.05,{ }^{* *} p<.01 .{ }^{1}$ Da gerichtete Zusammenhangshypothesen aufgestellt wurden, werden für die gekennzeichneten Indikatoren einseitige Signifikanztests verwendet. 
Tabelle 3: Einfluss individueller Stimmung auf die Bewertung des Zustands der Demokratie

\begin{tabular}{|c|c|c|c|c|c|c|c|c|c|}
\hline & \multicolumn{9}{|c|}{ Bewertung des Zustands der Demokratie } \\
\hline & ESS’06-I & \multicolumn{2}{|c|}{ ESS’06-II } & \multicolumn{2}{|c|}{ ESS’06-III } & \multicolumn{2}{|c|}{ ESS'12-I } & \multicolumn{2}{|c|}{ ESS'12-II } \\
\hline & & $\mathrm{b}$ & $\beta$ & $\mathrm{b}$ & $\beta$ & $\mathrm{b}$ & $\beta$ & $\mathrm{b}$ & $\beta$ \\
\hline positive Stimmung $^{1}$ & $0.45 * .11$ & $0.32 * *$ & .08 & $0.35 *$ & .08 & $0.37 * *$ & .11 & $0.28 * *$ & .08 \\
\hline negative Stimmung ${ }^{1}$ & $-0.55 *-.10$ & $-0.38 * *$ & -.07 & $-0.41 * *$ & -.08 & $-0.37 * *$ & -.08 & $-0.24 *$ & -.05 \\
\hline pos. Stimmg. * informiert ${ }^{1}$ & & & & $-0.14^{+}$ & -.07 & & & & \\
\hline neg. Stimmg. ${ }^{*}$ informiert $^{1}$ & & & & 0.23 & .06 & & & & \\
\hline Alter (in Jahren) & & $-0.01 * *$ & -.06 & $-0.01 * *$ & -.06 & & & $-0.01 *$ & -.05 \\
\hline Geschlecht (männlich) & & -0.05 & -.01 & -0.05 & -.01 & & & -0.01 & -.00 \\
\hline Bildung (in Jahren) & & -0.00 & -.00 & -0.00 & -.00 & & & 0.01 & .02 \\
\hline Arbeitslosigkeit & & $-0.49 * *$ & -.05 & $-0.48 *$ & -.05 & & & $-0.49 * *$ & -.05 \\
\hline HH.-Einkommen & & $0.11^{* * *}$ & .15 & $0.11 * *$ & .15 & & & $0.09 * *$ & .14 \\
\hline HH.-Eink.: k. Angabe & & $0.49 *$ & .08 & $0.50^{*}$ & .09 & & & $0.51^{* * *}$ & .08 \\
\hline pol. Zeitungslektüre & & $0.30^{* * *}$ & .10 & $0.33 * *$ & .11 & & & & \\
\hline pol. TV-Konsum & & -0.02 & -.01 & -0.02 & -.01 & & & 0.06 & .03 \\
\hline pol. Interesse & & -0.01 & -.00 & -0.01 & -.01 & & & $-0.15^{* *}$ & -.06 \\
\hline Bindung Reg.-Partei & & $1.05^{* *}$ & .21 & $1.04 * *$ & .21 & & & $0.70 * *$ & .14 \\
\hline politisch „links“ & & $-0.34 * *$ & -.06 & $-0.34 * *$ & -.06 & & & -0.09 & -.02 \\
\hline politisch „rechts“ & & 0.09 & .01 & 0.09 & .01 & & & -0.18 & -.03 \\
\hline Modellgüte $\left(\mathrm{R}^{2}\right)$ & .033 & .110 & & .11 & & .028 & & .075 & \\
\hline
\end{tabular}

Anmerkungen: Lineare Regressionsanalysen. $\mathrm{N}=2423$ (ESS 2006) und N = 2644 (ESS 2012). Signifikanzwerte: ${ }^{+} p<.10,{ }^{*} p<.05,{ }^{* *} p<.01$. 'Da gerichtete Zusammenhangshypothesen aufgestellt wurden, werden für die gekennzeichneten Indikatoren einseitige Signifikanztests verwendet. 
Tabelle 4: Einfluss individueller Stimmung auf das soziale Vertrauen

\begin{tabular}{|c|c|c|c|c|c|c|c|c|c|}
\hline & \multicolumn{9}{|c|}{ Soziales Vertrauen } \\
\hline & ESS'06-I & \multicolumn{2}{|c|}{ ESS’06-II } & \multicolumn{2}{|c|}{ ESS’06-III } & \multicolumn{2}{|c|}{ ESS'12-I } & \multicolumn{2}{|c|}{ ESS'12-II } \\
\hline & $\mathrm{b} \quad \beta$ & $\mathrm{b}$ & $\beta$ & $\mathrm{b}$ & $\beta$ & $\mathrm{b}$ & $\beta$ & $\mathrm{b}$ & $\beta$ \\
\hline positive Stimmung $^{1}$ & $0.34 * .09$ & $0.21 *$ & .05 & $0.21 *$ & .05 & $0.15^{*}$ & .04 & $0.13^{+}$ & .04 \\
\hline negative Stimmung ${ }^{1}$ & $-0.57 * *-.11$ & $-0.45^{* *}$ & -.09 & $-0.50^{* * *}$ & -.10 & $-0.61 * *$ & -.13 & $-0.44 * *$ & -.09 \\
\hline pos. Stimmg. "informiert ${ }^{1}$ & & & & 0.00 & .00 & & & & \\
\hline neg. Stimmg. ${ }^{*}$ informiert ${ }^{1}$ & & & & $0.27^{+}$ & .07 & & & & \\
\hline Alter (in Jahren) & & $-0.01 * *$ & -.08 & $-0.01 * *$ & -.08 & & & 0.00 & .01 \\
\hline Geschlecht (männlich) & & $-0.30 * *$ & -.06 & $-0.30 * *$ & -.07 & & & -0.10 & -.02 \\
\hline Bildung (in Jahren) & & $0.07^{* *}$ & .11 & $0.07^{* * *}$ & .11 & & & $0.10^{* *}$ & .16 \\
\hline Arbeitslosigkeit & & $-0.34^{+}$ & -.04 & $-0.34^{+}$ & -.04 & & & $-0.41 *$ & -.04 \\
\hline HH.-Einkommen & & $0.07^{*}$ & .10 & $0.07^{*}$ & .10 & & & $0.06 * *$ & .09 \\
\hline HH.-Eink.: k. Angabe & & $0.44 *$ & .08 & $0.44 *$ & .08 & & & $0.43 * *$ & .07 \\
\hline pol. Zeitungslektüre & & $0.18 * *$ & .07 & 0.03 & .01 & & & & \\
\hline pol. TV-Konsum & & $-0.11 *$ & -.05 & $-0.12 *$ & -.06 & & & -0.06 & -.03 \\
\hline pol. Interesse & & $-0.23^{* *}$ & -.09 & $-0.23 * *$ & -.09 & & & $-0.26^{* *}$ & -.09 \\
\hline Bindung Reg.-Partei & & 0.08 & .02 & 0.10 & .02 & & & 0.17 & .03 \\
\hline politisch „links“ & & -0.07 & -.01 & -0.07 & -.01 & & & 0.11 & .02 \\
\hline politisch „rechts“ & & -0.20 & -.03 & -0.21 & -.03 & & & $-0.35 * *$ & -.05 \\
\hline Modellgüte $\left(\mathrm{R}^{2}\right)$ & .029 & $.07 \xi$ & & .079 & & .024 & & .084 & \\
\hline
\end{tabular}

Anmerkungen: Lineare Regressionsanalysen. $\mathrm{N}=2449$ (ESS 2006) und N = 2659 (ESS 2012). Signifikanzwerte: ${ }^{+} p<.10,{ }^{*} p<.05,{ }^{* *} p<.01 .{ }^{1}$ Da gerichtete Zusammenhangshypothesen aufgestellt wurden, werden für die gekennzeichneten Indikatoren einseitige Signifikanztests verwendet. 
Tabelle 5: Einfluss individueller Stimmung auf das Vertrauen in das Parlament

\begin{tabular}{|c|c|c|c|c|c|c|c|c|c|}
\hline & \multicolumn{9}{|c|}{ Vertrauen in das Parlament } \\
\hline & ESS’06-I & \multicolumn{2}{|c|}{ ESS’06-II } & \multicolumn{2}{|c|}{ ESS’06-III } & \multicolumn{2}{|c|}{ ESS'12-I } & \multicolumn{2}{|c|}{ ESS'12-II } \\
\hline & b $\quad \beta$ & $\mathrm{b}$ & $\beta$ & $\mathrm{b}$ & $\beta$ & $\mathrm{b}$ & $\beta$ & $\mathrm{b}$ & $\beta$ \\
\hline positive Stimmung ${ }^{1}$ & $0.37 * 09$ & $0.23 * *$ & .06 & $0.26 * *$ & .07 & $0.14^{+}$ & .04 & 0.02 & .01 \\
\hline negative Stimmung ${ }^{1}$ & $-0.43 *-.08$ & $-0.27 *$ & -.05 & $-0.34 * *$ & -.06 & $-0.49 * *$ & -.10 & $-0.37 * *$ & -.07 \\
\hline pos. Stimmg. ${ }^{*}$ informiert ${ }^{1}$ & & & & $-0.15^{+}$ & -.08 & & & & \\
\hline neg. Stimmg. ${ }^{*}$ informiert ${ }^{1}$ & & & & $0.40 *$ & .10 & & & & \\
\hline Alter (in Jahren) & & $-0.01 *$ & -.06 & $-0.01 *$ & -.05 & & & $-0.02 * *$ & -.14 \\
\hline Geschlecht (männlich) & & -0.02 & -.01 & -0.03 & -.01 & & & $-0.17^{+}$ & -.04 \\
\hline Bildung (in Jahren) & & $0.04 * *$ & .06 & $0.04 * *$ & .07 & & & $0.06 * *$ & .09 \\
\hline Arbeitslosigkeit & & $-0.40^{*}$ & -.05 & $-0.40 *$ & -.04 & & & $-0.40^{*}$ & -.04 \\
\hline HH.-Einkommen & & $0.09 * *$ & .14 & $0.10^{* *}$ & .14 & & & $0.08 * *$ & .12 \\
\hline HH.-Eink.: k. Angabe & & $0.62 * *$ & .11 & $0.63^{* *}$ & .11 & & & $0.58 * *$ & .08 \\
\hline pol. Zeitungslektüre & & $0.29 * *$ & .10 & $0.24 *$ & .08 & & & & \\
\hline pol. TV-Konsum & & 0.04 & .02 & 0.03 & .02 & & & 0.06 & .03 \\
\hline pol. Interesse & & -0.04 & -.01 & -0.04 & -.02 & & & $-0.44 * *$ & -.15 \\
\hline Bindung Reg.-Partei & & $0.83^{* *}$ & .17 & $0.84 * *$ & .17 & & & $0.70 * *$ & .13 \\
\hline politisch „links“ & & $-0.32 * *$ & -.06 & $-0.32^{* * *}$ & -.06 & & & -0.03 & -.01 \\
\hline politisch „rechts“ & & -0.19 & -.03 & -0.19 & -.03 & & & -0.03 & -.01 \\
\hline Modellgüte $\left(\mathrm{R}^{2}\right)$ & .023 & .091 & & .093 & & .015 & & .097 & \\
\hline
\end{tabular}

Anmerkungen: Lineare Regressionsanalysen. $\mathrm{N}=2420$ (ESS 2006) und N = 2640 (ESS 2012). Signifikanzwerte: ${ }^{+} p<.10,{ }^{*} p<.05,{ }^{* *} p<.01 .{ }^{1}$ Da gerichtete Zusammenhangshypothesen aufgestellt wurden, werden für die gekennzeichneten Indikatoren einseitige Signifikanztests verwendet 